\title{
ON SIMPLE LEFT, RIGHT AND TWO-SIDED IDEALS OF AN ORDERED SEMIGROUP HAVING A KERNEL
}

\author{
Thawhat Changphas
}

\begin{abstract}
The intersection of all two-sided ideals of an ordered semigroup, if it is non-empty, is called the kernel of the ordered semigroup. A left ideal $L$ of an ordered semigroup $(S, \cdot, \leq)$ having a kernel $I$ is said to be simple if $I$ is properly contained in $L$ and for any left ideal $L^{\prime}$ of $(S, \cdot, \leq)$, $I$ is properly contained in $L^{\prime}$ and $L^{\prime}$ is contained in $L$ imply $L^{\prime}=L$. The notions of simple right and two-sided ideals are defined similarly. In this paper, the author characterize when an ordered semigroup having a kernel is the class sum of its simple left, right and two-sided ideals. Further, the structure of simple two-sided ideals will be discussed.
\end{abstract}

\section{Introduction}

The intersection of all two-sided ideals of a semigroup, if it is non-empty, is called the kernel [5] of the semigroup.

Let $S$ be a semigroup having a kernel $I$. A left ideal $L$ of $S$ is said to be simple if $I \subset L$, (The symbol $\subset$ stands for proper inclusion of sets.) and for any left ideal $L^{\prime}$ of $S$, if $I \subset L^{\prime} \subseteq L$, then $L^{\prime}=L$. The notions of simple right and twosided ideals are defined similarly. These concepts were introduced and studied by Schwarz [6]. Indeed, the author characterized when a semigroup having a kernel is the class sum of its simple left, right or two-sided ideals. Further, the structure of simple two-sided ideals of some semigroups was investigated.

The purpose of this paper is to extend Schwarz's results to ordered semigroups. Section 2 recalls some certain definitions and results used throughout this paper. In Section 3, we introduce the concepts of simple left, right and twosided ideals of an ordered semigroup having a kernel. Necessary and sufficient conditions for an ordered semigroup having a kernel to be the class sum of its simple left, right or two-sided ideals are given (Theorems 3.9-3.11). Further, we obtain important corollaries using annihilators of an ordered semigroup. Sections 4-5 investigate the structure of simple two-sided ideals of an ordered semigroup having a kernel.

Received November 14, 2013.

2010 Mathematics Subject Classification. Primary 06F05.

Key words and phrases. semigroup, simple ordered semigroup, kernel, simple ideal, ideal, annihilator, radical, $I$-potent. 


\section{Preliminaries}

A semigroup $(S, \cdot)$ together with a partial order $\leq$ that is compatible with the semigroup operation, meaning that, for any $x, y, z$ in $S$,

$$
x \leq y \text { implies } z x \leq z y \text { and } x z \leq y z,
$$

is called a partially ordered semigroup (or simply an ordered semigroup) [1]. For any $a, b$ in $S$, the symbol $a \not \leq b$ means $a \leq b$ is false.

Let $(S, \cdot, \leq)$ be an ordered semigroup. If $A$ and $B$ are non-empty subsets of $S$, then we write

$$
\begin{gathered}
A B=\{x y \in S \mid x \in A, y \in B\}, \\
(A]=\{x \in S \mid x \leq a \text { for some } a \in A\} .
\end{gathered}
$$

For an element $a$ in $S$, we write $(\{a\} \cup A]$ as $(a \cup A]$. It is routine matters to verify that the following hold:

(1) $A \subseteq B$ implies $(A] \subseteq(B]$;

(2) $((A]]=(A]$;

(3) $(A](B] \subseteq(A B]$;

(4) $((A](B]]=(A B]$.

The concepts of left, right and two-sided ideals of an ordered semigroup are defined by Kehayopulu [2].

Definition. Let $(S, \cdot, \leq)$ be an ordered semigroup. A non-empty subset $A$ of $S$ is called a left (respectively, right) ideal of an ordered $\operatorname{semigroup}(S, \cdot, \leq)$ if it satisfies:

(i) $S A \subseteq A$ (respectively, $A S \subseteq A$ );

(ii) for any $x$ in $A$ and $y$ in $S$, if $y \leq x$, then $y \in A$, or equivalently, $A=(A]$. If $A$ is both a left and a right ideal of $S$, then $A$ is called a two-sided ideal (or an ideal) of $S$. hold:

Let $(S, \cdot, \leq)$ be an ordered semigroup. It is easy to see that the following

(1) If $A$ is a left ideal of $S$ and $B$ a right ideal of $S$, then $(A B]$ is a two-sided ideal of $S$.

(2) If $A$ and $B$ are left (respectively, right, two-sided) ideals of $S$, then $A \cup B$ and $A \cap B$ (if it is non-empty) are left (respectively, right, two-sided) ideals of $S$.

(3) If $A$ is a non-empty subset of $S$, then the principal left (respectively, right, two-sided) ideal of $S$ generated by $A$ is

$$
(A \cup S A] \text { (respectively, }(A \cup A S],(A \cup S A \cup A S \cup S A S]) \text {. }
$$

(4) If $A$ is a non-empty subset of $S$, then

( $S A]$ (respectively, $(A S],(S A S])$

is a left (respectively, right, two-sided) ideal of $S$. 
Definition. The intersection of all two-sided ideals of an ordered semigroup $(S, \cdot, \leq)$, if it is non-empty, is called a kernel [4] of $S$.

Note that if $I$ is a kernel of an ordered semigroup $(S, \cdot, \leq)$, then $\left(I^{2}\right] \subseteq I$; hence $\left(I^{2}\right]=I$.

Example 2.1 ([4]). Consider an ordered semigroup $(S, \cdot, \leq)$ where the multiplication and the relation are defined by:

and

\begin{tabular}{c|ccccc}
$\cdot$ & $a$ & $b$ & $c$ & $d$ & $e$ \\
\hline$a$ & $a$ & $b$ & $a$ & $a$ & $a$ \\
$b$ & $a$ & $b$ & $a$ & $a$ & $a$ \\
$c$ & $a$ & $b$ & $a$ & $a$ & $a$ \\
$d$ & $a$ & $b$ & $a$ & $a$ & $a$ \\
$e$ & $a$ & $b$ & $a$ & $a$ & $e$
\end{tabular}

$\leq=\{(a, a),(a, b),(a, e),(b, b),(c, b),(c, c),(c, e),(d, b),(d, d),(d, e),(e, e)\}$.

The right ideals of $S$ are $\{a, b, c, d\}$ and $S$.

The left ideals of $S$ are $\{a\},\{a, c\},\{a, d\},\{a, c, d\},\{a, b, c, d\},\{a, c, d, e\}$ and $S$.

Then the two-sided ideals of $S$ are $\{a, b, c, d\}$ and $S$; hence the kernel of $S$ is $\{a, b, c, d\}$.

3. The class sum of simple left (respectively, right, twi-sided) ideals

Definition. Let $(S, \cdot, \leq)$ be an ordered semigroup having a kernel $I$. A left ideal $L$ of $S$ is called a simple left ideal of $S$ if $L$ satisfies the following conditions:

(i) $I \subset L \subseteq S$;

(ii) if $L^{\prime}$ is a left ideal of $S$ such that $I \subset L^{\prime} \subseteq L$, then $L^{\prime}=L$.

For simple right ideals of $S$ are defined similarly.

Example 3.1 ([3]). Consider an ordered semigroup $(S, \cdot, \leq)$ where the multiplication and the relation are defined by:

and

\begin{tabular}{c|lllll}
$\cdot$ & $a$ & $b$ & $c$ & $d$ & $e$ \\
\hline$a$ & $b$ & $d$ & $a$ & $b$ & $e$ \\
$b$ & $d$ & $b$ & $b$ & $d$ & $e$ \\
$c$ & $d$ & $b$ & $c$ & $d$ & $e$ \\
$d$ & $b$ & $d$ & $d$ & $b$ & $e$ \\
$e$ & $e$ & $e$ & $e$ & $e$ & $e$
\end{tabular}

$$
\leq=\{(a, a),(a, b),(a, e),(b, b),(c, b),(c, c),(c, e),(d, b),(d, d),(d, e),(e, e)\} .
$$

The right ideals of $S$ are $\{b, d, e\},\{a, b, d, e\},\{b, c, d, e\}$ and $S$.

The left ideals of $S$ are $\{b, d, e\},\{a, b, d, e\}$ and $S$.

Then the two-sided ideals of $S$ are $\{b, d, e\},\{a, b, d, e\}$ and $S$; hence the kernel of $S$ is $\{b, d, e\}$. 
It is easy to see that there are three simple right ideals of $S:\{a, b, d, e\}$, $\{b, c, d, e\}$ and $S$, and there are two simple left ideals of $S:\{a, b, d, e\}$ and $S$.

We now investigate some properties of simple left ideals of an ordered semigroup having a kernel.

Theorem 3.2. Let $(S, \cdot, \leq)$ be an ordered semigroup having a proper kernel $I$. If $L_{1}$ and $L_{2}$ are simple left ideals of $S$, then $L_{1}=L_{2}$ or $L_{1} \cap L_{2}=I$.

Proof. If $L_{1}$ and $L_{2}$ are simple left ideals of $S$, then $I \subseteq L_{1} \cap L_{2} \subseteq L_{1}$ and $I \subseteq L_{1} \cap L_{2} \subseteq L_{2}$. If $I \subset L_{1} \cap L_{2}$, then $L_{1}=L_{1} \cap L_{2}$ and $L_{2}=L_{1} \cap \bar{L}_{2}$; hence $L_{1}=L_{2}$.

Theorem 3.3. Let $(S, \cdot, \leq)$ be an ordered semigroup having a kernel $I$. If $L$ is a simple left ideal of $S$, then $(L I]=(I L]=I$.

Proof. Assume that $L$ is a simple left ideal of $S$. Then $I \subseteq(L I] \subseteq I$ since $(L I]$ is a two-sided ideal of $S$; hence $(L I]=I$. We have

$$
I=\left(I^{2}\right] \subseteq(I L] \subseteq(I S] \subseteq I .
$$

Thus $(I L]=I$.

Theorem 3.4. Let $(S, \cdot, \leq)$ be an ordered semigroup having a proper kernel $I$. If $L_{1}$ and $L_{2}$ are simple left ideals of $S$, then $\left(L_{1} L_{2}\right]=I$ or $\left(L_{1} L_{2}\right]=L_{2}$.

Proof. If $L_{1}$ and $L_{2}$ are simple left ideals of $S$, then

$$
I=\left(I^{2}\right] \subseteq\left(L_{1} L_{2}\right] \subseteq\left(S L_{2}\right] \subseteq L_{2},
$$

and so $\left(L_{1} L_{2}\right]=I$ or $\left(L_{1} L_{2}\right]=L_{2}$.

The following is a consequence of Theorem 3.4:

Corollary 3.5. If $L$ is a simple left ideal of an ordered semigroup $(S, \cdot, \leq)$ having a proper kernel $I$, then $\left(L^{2}\right]=I$ or $\left(L^{2}\right]=L$.

Definition. Let $(S, \cdot, \leq)$ be an ordered semigroup having a kernel $I$. A twosided ideal $A$ of $S$ is said to be a simple two-sided ideal of $S$ if $A$ satisfies the following conditions:

(i) $I \subset A \subseteq S$;

(ii) if $A^{\prime}$ is a two-sided ideal of $S$ such that $I \subset A^{\prime} \subseteq A$, then $A^{\prime}=A$.

Theorem 3.6. Let $(S, \cdot, \leq)$ be an ordered semigroup having a kernel I. If $A_{1}$ and $A_{2}$ are two different simple two-sided ideals of $S$, then

$$
\left(A_{1} A_{2}\right]=A_{1} \cap A_{2}=I \text {. }
$$

Proof. As in the proof of Theorem 3.2, we have $A_{1} \cap A_{2}=I$. Since $I=\left(I^{2}\right] \subseteq$ $\left(A_{1} A_{2}\right] \subseteq A_{1}$, so $I \subseteq A_{1}$. Similarly, $I \subseteq A_{2}$, and thus

$$
I \subseteq\left(A_{1} A_{2}\right] \subseteq A_{1} \cap A_{2}=I .
$$

Hence $\left(A_{1} A_{2}\right]=I$. 
This is a consequence of Theorem 3.6:

Corollary 3.7. If $A$ is a simple ideal of an ordered semigroup $(S, \cdot, \leq)$ having a kernel $I$, then $\left(A^{2}\right]=A$ or $\left(A^{2}\right]=I$.

An ordered semigroup $(S, \cdot, \leq)$ having a kernel is said to be the class sum of its simple left ideals if $S=\bigcup_{\alpha \in \Lambda} A_{\alpha}$ for some an indexed family $\left\{A_{\alpha}: \alpha \in \Lambda\right\}$ of simple left ideals of $S$. $S$ is the class sum of its simple right ideals or of its simple two-sided ideals are defined similarly.

Theorem 3.8. Let $(S, \cdot, \leq)$ be an ordered semigroup having a proper kernel I. If $S$ is the class sum of its simple left (respectively, right, two-sided) ideals, then this decomposition is uniquely determined.

Proof. Assume that $S$ is the class sum of its simple two-sided ideals:

$$
S=\bigcup_{\alpha \in \Lambda} A_{\alpha} \text { and } S=\bigcup_{\beta \in \Lambda^{\prime}} B_{\beta},
$$

where $A_{\alpha}$ and $B_{\beta}$ are simple two-sided ideals of $S$. We show that, for each $\beta \in \Lambda^{\prime}, B_{\beta} \subseteq A_{\alpha}$ for some $\alpha \in \Lambda$. Let $\beta \in \Lambda^{\prime}$. Then

$$
B_{\beta}=S \cap B_{\beta}=\bigcup_{\alpha \in \Lambda}\left(A_{\alpha} \cap B_{\beta}\right) .
$$

We have, for each $\alpha \in \Lambda, A_{\alpha} \cap B_{\beta}=I$ or $A_{\alpha} \cap B_{\beta}=A_{\alpha}=B_{\beta}$. Since $B_{\beta}$ is a simple two-sided ideal of $S$, there exists $\alpha \in \Lambda$ such that $A_{\alpha} \cap B_{\beta}=A_{\alpha}=B_{\beta}$. Similarly, for any $\alpha \in \Lambda$, there exists $\beta \in \Lambda^{\prime}$ such that $B_{\beta}=A_{\alpha}$. The other two cases are proved similarly.

Theorem 3.9. Let $(S, \cdot, \leq)$ be an ordered semigroup having a proper kernel I. Then $S$ is the class sum of its simple left ideals if and only if the following condition holds: if $a, b$ are in $S \backslash I$ such that $b \not \leq a$ and $a \leq x b$ for some $x$ in $S$, then $b \leq x^{\prime} a$ for some $x^{\prime}$ in $S \backslash I$.

Proof. Assume that $S$ is the class sum of its simple left ideals. To show that the condition holds, let $a, b$ be elements of $S \backslash I$ such that $b \not \leq a$ and $a \leq x b$ for some $x$ in $S$. By assumption, there exist simple left ideals $L_{a}$ containing $a$ and $L_{b}$ containing $b$. Since $I \subset I \cup(a \cup S a] \subseteq L_{a}$, we have $L_{a}=I \cup(a \cup S a]$. Similarly, $L_{b}=I \cup(b \cup S b]$. By Theorem 3.2, $L_{a} \cap L_{b}=I$ or $L_{a}=L_{b}$. If $L_{a} \cap L_{b}=I$, then, by $a \leq x b$, we get $a \in L_{a} \cap L_{b}$, giving $a \in I$. This is a contradiction. Thus $L_{a}=L_{b}$, i.e., $I \cup(a \cup S a]=I \cup(b \cup S b]$. Since $b \not \leq a$, there exists $x^{\prime}$ in $S$ such that $b \leq x^{\prime} a$. If $x^{\prime} \in I$, then $b \in I$. This is a contradiction. Thus $x^{\prime} \in S \backslash I$.

Conversely, assume that the condition holds. To show that $S$ is the class sum of its simple left ideals, we show that every element $a$ in $S \backslash I$ is an element of a simple left ideal of $S$. Let $a$ be an element of $S \backslash I$. We prove that $I \cup(a \cup S a]$ is a simple left ideal of $S$, and then the proof is completed. Clearly, $I \subset I \cup(a \cup S a]$ and $I \cup(a \cup S a]$ is a left ideal containing $a$. Let $L$ be a left ideal of $S$ such that $I \subset L \subset I \cup(a \cup S a]$, so that there exists $b$ in $L \backslash I$ and 


$$
I \subset I \cup(b \cup S b] \subseteq L \subset I \cup(a \cup S a] .
$$

Since $a \not \leq b$ and $b \leq x a$ for some $x$ in $S$, it follows by assumption that there exists $x^{\prime}$ in $S \backslash I$ such that $a \leq x^{\prime} b$. This implies $a \in I \cup(b \cup S b]$. Then $I \cup(a \cup S a] \subseteq I \cup(b \cup S b]$, and hence $L=I \cup(a \cup S a]$. This is a contradiction.

The following theorem is proved dually:

Theorem 3.10. Let $(S, \cdot, \leq)$ be an ordered semigroup having a proper kernel $I$. Then $S$ is the class sum of its simple right ideals if and only if the following condition holds: if $a, b$ are in $S \backslash I$ such that $b \not \leq a$ and $a \leq b y$ for some $y$ in $S$, then $b \leq a y^{\prime}$ for some $y^{\prime}$ in $S \backslash I$.

An ordered semigroup $(S, \cdot, \leq)$ is said to be the direct sum of two-sided ideals of $S$ if $S$ is the class sum of its simple two-sided ideals. It is clear that if an ordered semigroup $(S, \cdot, \leq)$ having a kernel $I$ is the direct sum of two-sided ideals $A_{\alpha}(\alpha \in \Lambda)$ of $S$, then $\left(A_{\alpha} A_{\beta}\right]=I$ for all $\alpha \neq \beta$.

Theorem 3.11. Let $(S, \cdot, \leq)$ be an ordered semigroup having a kernel I. Then $S$ is the direct sum of two-sided ideals of $S$ if and only if the following condition holds: if $a, b$ are in $S \backslash I$ such that $b \not \leq a$ and $a \leq b x_{1}$ for some $x_{1}$ in $S$ or $a \leq x_{2} b$ for some $x_{2}$ in $S$ or $a \leq x_{3} b x_{4}$ for some $x_{3}, x_{4}$ in $S$, then $b \leq a x_{1}^{\prime}$ for some $x_{1}^{\prime}$ in $S$ or $a \leq x_{2}^{\prime} b$ for some $x_{2}^{\prime}$ in $S$ or $b \leq x_{3}^{\prime} a x_{4}^{\prime}$ for some $x_{3}^{\prime}, x_{4}^{\prime}$ in $S$.

Proof. Assume that $S$ is the direct sum of two-sided ideals of $S$, i.e., $S$ is the class sum of simple two-sided ideals of $S$. To show that the condition holds, let $a, b$ be elements of $S \backslash I$ such that $b \not \leq a$ and $a$ can be written in at least one of the forms $a \leq b x_{1}$ for some $x_{1}$ in $S$ or $a \leq x_{2} b$ for some $x_{2}$ in $S$ or $a \leq x_{3} b x_{4}$ for some $x_{3}, x_{4}$ in $S$. By assumption, there is a simple two-sided ideal $M_{a}$ containing $a$. Since $I \subset I \cup(a \cup a S \cup S a \cup S a S] \subseteq M_{a}$, we have $M_{a}=I \cup(a \cup a S \cup S a \cup S a S]$. It follows that $M_{a}=(a \cup a S \cup S a \cup S a S]$ since $I \subseteq(S a S]$. Similarly, there is a simple two-sided ideal $M_{b}$ containing $b$ such that $M_{b}=(b \cup b S \cup S b \cup S b S]$. By Theorem 3.2, $M_{a} \cap M_{b}=I$ or $M_{a}=M_{b}$. Since $a \leq b x_{1}$ or $a \leq x_{2} b$ or $a \leq x_{3} b x_{4}$, we have $M_{a}=M_{b}$. This says $(a \cup a S \cup S a \cup S a S]=(b \cup b S \cup S b \cup S b S]$. Since $b \not \leq a$, so $b$ can be written in at least one of the forms $b \leq a x_{1}^{\prime}$ for some $x_{1}^{\prime}$ in $S$ or $a \leq x_{2}^{\prime} b$ for some $x_{2}^{\prime}$ in $S$ or $b \leq x_{3}^{\prime} a x_{4}^{\prime}$ for some $x_{3}^{\prime}, x_{4}^{\prime}$ in $S$ as required.

Conversely, assume that the condition holds. We have to show that $S$ is the class sum of simple two-sided ideals of $S$, i.e., for every element $a$ in $S \backslash I$, there exists a simple two-sided ideal $M_{a}$ containing $a$. Let $a$ be an element of $S \backslash I$. We set $M_{a}=(a \cup S a \cup a S \cup S a S]$. Suppose that there exists a two-sided ideal $M$ of $S$ such that $I \subset M \subset M_{a}$, so that there exist $b$ in $M \backslash I$ and a simple two-sided ideal $M_{b}$ containing $b$ such that $I \subset M_{b} \subseteq M \subset M_{a}$. We have $b \not \leq a$. Since $b$ is in $M_{a}$, it follows that $b \in(S a]$ or $b \in(a S]$ or $b \in(S a S]$. Using the assumption, we get $a \in(S b]$ or $a \in(b S]$ or $a \in(S b S]$. If $a \in(S b]$, then $(a S] \subseteq(S b S]$, and hence $(S a] \subseteq\left(S^{2} b\right] \subseteq(S b]$. Thus $(S a S] \subseteq(S b S]$ and 
so $M_{a} \subseteq M_{b}$. This is a contradiction. Similarly, if $a \in(b S]$ or $a \in(S b S]$, then $M_{a} \subseteq M_{b}$. This is a contradiction. This completes the proof.

Definition. Let $(S, \cdot, \leq)$ be an ordered semigroup having a kernel $I$. The right annihilator of $S$, denoted by $I_{r}$, is defined to be the set of all elements $a$ in $S$ such that $(S a] \subseteq I$. Dually, the left annihilator of $S$, denoted by $I_{l}$, is the set of all elements $a$ in $S$ such that $(a S] \subseteq I$.

Theorem 3.12. The following hold for an ordered semigroup $(S, \cdot, \leq)$ having a kernel $I$.

(1) $I \subseteq I_{r}, I \subseteq I_{l}$;

(2) $I_{r}, I_{l}$ are ideals of $S$;

(3) $\left(S I_{r}\right]=I,\left(I_{l} S\right]=I$;

(4) $\left(I_{r} I_{r}\right]=I,\left(I_{l} I_{l}\right]=I$;

(5) $\left(I_{l} I_{r}\right]=I$.

Proof. (1) follows by $(S I]=(I S]=I$.

(2) We prove that $I_{l}$ is an ideal of $S$. For $I_{r}$ is an ideal of $S$ is proved similarly. Let $a$ be an element of $I_{l}$ and $x$ an element of $S$. We have

$$
\begin{aligned}
& ((x a) S]=(x(a S)] \subseteq(x I] \subseteq I, \\
& ((a x) S]=(a(x S)] \subseteq(a S] \subseteq I .
\end{aligned}
$$

If $x \leq a$, then $(x S] \subseteq(a S] \subseteq I$, and hence $x$ is in $I_{l}$. Thus $I_{l}$ is an ideal of $S$.

(3) follows by (2).

(4) It is clear that $I \subseteq\left(I_{l} I_{l}\right]$. For the reverse inclusion we have $\left(I_{l} I_{l}\right] \subseteq$ $\left(I_{l} S\right] \subseteq I$, and so $I=\left(I_{l} I_{l}\right]$. Similarly, $\left(I_{r} I_{r}\right]=I$.

(5) This follows by the fact that $\left(I_{l} I_{r}\right]$ is an ideal of $S$.

Corollary 3.13. Let $(S, \cdot, \leq)$ be an ordered semigroup having a kernel I. If $S$ is the class sum of its simple left ideals, then every element a in $S \backslash I_{r}$ there exists e in $S \backslash I_{l}$ such that $a \leq e a$.

Proof. Assume that $S$ is the class sum of its simple left ideals. Let $a$ be an element of $S \backslash I_{r}$; then $a \in L_{a}$ for some simple left ideal $L_{a}$ of $S$. Since $a \in S \backslash I_{r}$, we have $a \in S \backslash I$, and so $I \subset I \cup(a \cup S a] \subseteq L_{a}$. It follows that $L_{a}=I \cup(a \cup S a]$. Since $a \in S \backslash I_{r}$, we obtain $(S a] \neq I$, and so $I \subset(S a] \subseteq L_{a}$. It follows that $I \cup(a \cup S a]=(a S]$. Thus $a \leq a e$ for some $e$ in $S$. If $e \in I_{l}$, then $a \leq a e \in I$. This is a contradiction. Hence $e \in S \backslash I_{l}$.

The following corollary is proved dually:

Corollary 3.14. Let $(S, \cdot, \leq)$ be an ordered semigroup with a kernel I. If $S$ is the class sum of its simple right ideals, then every a in $S \backslash I_{l}$ there exists $f$ in $S \backslash I_{r}$ such that $a \leq a f$.

Definition. Let $(S, \cdot, \leq)$ be an ordered semigroup having a kernel $I$. The set of all $a$ in $S$ such that $(S a S]=I$ will be denoted by $I_{0}$. 
Theorem 3.15. The following hold for an ordered semigroup $(S, \cdot, \leq)$ having a kernel $I$.

(1) $I_{0}$ is an ideal of $S$;

(2) $\left(I_{0}^{2}\right] \subseteq I_{r},\left(I_{0}^{2}\right] \subseteq I_{l}$;

(3) $\left(I_{0}^{3}\right]=I$.

Proof. (1) Let $a$ be an element of $I_{0}$ and $x$ an element of $S$. We have $(S a x S] \subseteq$ $(S a S]=I$ and $(S x a S] \subseteq(S a S]=I$. If $x \leq a$, then $(S x S] \subseteq(S a S]=I$, and hence $x \in I_{0}$. Thus $I_{0}$ is an ideal of $S$.

(2) Since $\left(I_{0}^{2}\right]$ is an ideal of $S, I \subseteq\left(I_{0}^{2}\right]$. We have $\left(I_{0}^{2} S\right] \subseteq\left(S I_{0} S\right] \subseteq I$. Then $\left(I_{0}^{2}\right] \subseteq I_{l}$. Similarly, $\left(I_{0}^{2}\right] \subseteq I_{r}$.

(3) Since $\left(I_{0}^{3}\right]$ is an ideal of $S$, so $I \subseteq\left(I_{0}^{3}\right]$. We have $\left(I_{0}^{3}\right] \subseteq\left(S I_{0} S\right] \subseteq I$. Then $\left(I_{0}^{3}\right]=I$.

Corollary 3.16. Let $(S, \cdot, \leq)$ be an ordered semigroup having a kernel I. If $S$ is the direct sum of two-sided ideals of $S$, then every a in $S \backslash I_{0}$ there exist $e$ in $S \backslash I_{l}$ and $f$ in $S \backslash I_{r}$ such that $a \leq e a f$.

Proof. Assume that $S$ is the direct sum of two-sided ideals of $S$. Let $a$ be an element of $S \backslash I_{0}$. Then $a \in S \backslash I$ and there exists a simple two-sided ideal $M_{a}$ containing $a$. We have $M_{a}=I \cup(a \cup S a \cup S a \cup S a S]$ since $I \subset(a \cup S a \cup a S \cup S a S] \subseteq$ $M_{a}$. We now consider a two-sided ideal $(S a S]$ of $S$. Since $a \in S \backslash I_{0}$, we obtain $(S a S] \neq I$, and so $I \subset(S a S] \subseteq M_{a}$. Thus $(S a S]=I \cup(a \cup S a \cup a S \cup S a S]$. Hence $a \leq e a f$ for some $e, f$ in $S$. If $e \in I_{l}$ or $f \in I_{r}$, then eaf $\in I$, and hence $a \in I$. This is a contradiction.

\section{Simple two-sided ideals of an ordered semigroup having a kernel}

Theorem 4.1. Let $(S, \cdot, \leq)$ be an ordered semigroup with a kernel I. If $M$ is a two-sided ideal of $S$, then $M$ has a kernel $I$.

Proof. Assume that $M$ is a two-sided ideal of $S$. Since $I M \subseteq I S \subseteq I$ and $M I \subseteq S I \subseteq I$, we have $I$ is a two-sided ideal of $M$. Let $A$ be any two-sided ideal of $M$ such that $A \subseteq I$. Since $A M \subseteq A$ and $M A \subseteq A$, it follows that $(M A M] \subseteq A$. Since $(M \bar{A} M]$ is a two-sided ideal of $S$, so $I \subseteq(M A M]$, and hence $I \subseteq(M A M] \subseteq A$. By $A \subseteq I$ follows $A=I$.

The following is a consequence of Theorem 4.1:

Corollary 4.2. Every simple left (respectively, right, two-sided) ideal of a twosided ideal $M$ of an ordered semigroup $(S, \cdot, \leq)$ having a kernel I contains the kernel $I$.

Lemma 4.3. Let $(S, \cdot, \leq)$ be an ordered semigroup with a kernel I. If $M$ is a simple two-sided ideal of $S$ such that $\left(M^{2}\right] \neq I$, then $M$ contains no left ideal $L$ of $S$ not entirely in $I$ and $\left(L^{2}\right] \subseteq I$. 
Proof. Assume that $M$ is a simple two-sided ideal of $S$ such that $\left(M^{2}\right] \neq I$. Suppose $M$ contains a left ideal $L$ of $S$ not entirely in $I$ and $\left(L^{2}\right] \subseteq I$. Since $L \cup(L S]$ is a two-sided ideal of $S$ such that $I \subset L \cup(L S] \subseteq M$, so $M=L \cup(L S]$. We have

$$
I=\left(I^{2}\right] \subseteq((L \cup(L S])(L \cup(L S])] \subseteq\left(L^{2}\right] \subseteq I .
$$

This says $\left((L \cup(L S])^{2}\right]=I$. Then $\left(M^{2}\right]=I$. This is a contradiction.

Theorem 4.4. Let $(S, \cdot, \leq)$ be an ordered semigroup having a kernel I. If $M$ is a simple two-sided ideal of $S$ such that $\left(M^{2}\right] \neq I$, then $M$ is a simple ordered semigroup (with a kernel $I$ ).

Proof. Assume that $M$ is a simple two-sided ideal of $S$ such that $\left(M^{2}\right] \neq I$. To show that $M$ is a simple ordered semigroup, we let $B$ be a two-sided ideal of $M$ such that $I \subset B \subset M$. If $B$ is a two-sided ideal of $S$, then we get a contradiction; hence the proof is completes. Since $(M B M] \subseteq B \subset M$ and $(M B M]$ is a two-sided ideal of $S$, we have $(M B M]=I$.

Moreover, $(M B] \subseteq I$ and $(B M] \subseteq I$. To see this, since

$$
((M B](M B]]=(M B M B] \subseteq(I B] \subseteq(I]=I,
$$

we have $(M B] \subseteq I$ by Lemma 4.3. Similarly, $(M B] \subseteq I$.

We have $(S B] \subseteq I$ and $(S B] \subseteq I$. In fact, since $(S B S] \subseteq(S M S] \subseteq M$ and $(S B S]$ is a two-sided of $S$, it follows that $(S B S]=I$ or $(S B S]=M$.

Case 1: $(S B S]=I$. Since

$$
((S B](S B]]=(S B S B] \subseteq(I B] \subseteq(I]=I
$$

and $(S B]$ is a two-sided ideal of $S$, so $(S B] \subseteq I$ by Lemma 4.3. Similarly, $(B S] \subseteq I$.

Case $2:(S B S]=M$. We have

$$
\begin{aligned}
& ((S B](S B]]=(S B S B] \subseteq(M B] \subseteq I, \\
& ((B S](B S]]=(B S B S] \subseteq(B M] \subseteq I .
\end{aligned}
$$

Then $(S B] \subseteq I$ and $(B S] \subseteq I$ by Lemma 4.3 .

Hence $S B \subseteq B$ and $B S \subseteq B$ as required.

Theorem 4.5. Let $(S, \cdot, \leq)$ be an ordered semigroup with a kernel I. If $M$ is a two-sided ideal of $S$ such that $\left(M^{2}\right] \neq I$, then the following hold:

(1) if $L^{M}$ is a simple left ideal of $M$, then $L^{M}$ is a (simple) left ideal of $S$;

(2) if $L^{S}$ is a simple left ideal of $S$ such that $I \subset M \cap L^{S}$, then $L^{S}$ is a simple left ideal of $M$.

Proof. (1) Assume that $L^{M}$ is a simple left ideal of $M$. Since $I \subseteq\left(M L^{M}\right] \subseteq$ $L^{M}$, it follows that $\left(M L^{M}\right]=I$ or $\left(M L^{M}\right]=L^{M}$. If $\left(M L^{M}\right]=I$, then

$$
\left(\left(M L^{M}\right]\left(M L^{M}\right]\right]=\left(M L^{M} M L^{M}\right] \subseteq\left(M L^{M}\right]=I .
$$


This is impossible by Lemma 4.3. Thus $\left(M L^{M}\right]=L^{M}$. This implies that

$$
S L^{M}=S\left(M L^{M}\right] \subseteq\left(S M L^{M}\right] \subseteq\left(M L^{M}\right]=L^{M} .
$$

Hence $L^{M}$ is a left ideal of $S$.

(2) Assume that $L^{S}$ is a simple left ideal of $S$ such that $I \subset M \cap L^{S}$. Since $I \subset M \cap L^{S} \subseteq L^{S}$, so $M \cap L^{S}=L^{S}$; hence $L^{S} \subseteq M$. By $M L^{S} \subseteq S L^{S} \subseteq L^{S}$ follows $L^{S}$ is a left ideal of $M$.

To show that $L^{S}$ is simple (in $M$ ), let $B^{M}$ be a left ideal of $M$ such that $I \subset B^{M} \subset L^{S}$. We have $\left(M B^{M}\right]$ is a left ideal of $S$ and $\left(M B^{M}\right] \subseteq B^{M} \subset L^{S}$. Then $\left(M B^{M}\right]=I$. Since $\left(S B^{M}\right]$ is a left ideal of $S$ such that $\left(S B^{M}\right] \subseteq$ $\left(S L^{S}\right] \subseteq L^{S}$, it follows that $\left(S B^{M}\right]=I$ or $\left(S B^{M}\right]=L^{S}$. If $\left(S B^{M}\right]=I$, then $S B^{M} \subseteq\left(S B^{M}\right]=I \subset B^{M}$; hence $B^{M}$ is a left ideal of $S$. This is impossible. If $\left(S B^{\bar{M}}\right]=L^{S}$, then

$$
\left(L^{S} L^{S}\right]=\left(S B^{M} S B^{M}\right] \subseteq\left(S M S B^{M}\right] \subseteq\left(M B^{M}\right]=I .
$$

This is a contradiction. This completes the proof.

\section{Ordered semigroups without a proper radical}

Let $(S, \cdot \leq)$ be an ordered semigroup having a kernel $I$. We have a sequence of two-sided ideals of $S$ as follows:

$$
S \supseteq\left(S^{2}\right] \supseteq\left(S^{3}\right] \supseteq \cdots .
$$

It follows that either (1) or (2) holds:

(1) $I \subset\left(S^{n}\right]$ for all positive integers $n$;

(2) there exists a positive integer $n_{0}$ such that $\left(S^{n}\right]=I$ for all positive integers $n \geq n_{0}$.

This leads to the following definition:

Definition. Let $(S, \cdot, \leq)$ be an ordered semigroup having a kernel $I$. A nonempty subset $A$ of $S$ is said to be $I$-potent if there exists a positive integer $n$ such that $\left(A^{n}\right] \subseteq I$.

Note that a left ideal $L$ of an ordered semigroup $(S, \cdot, \leq)$ having a kernel $I$ is $I$-potent if there exists a positive integer $n$ such that $\left(L^{n}\right] \subseteq I$. A two-sided ideal $A$ of $S$ is $I$-potent if there exists a positive integer $m$ such that $\left(A^{m}\right]=I$.

Theorem 5.1. Let $(S, \cdot, \leq)$ be an ordered semigroup having a kernel $I$. If $L_{1}$ and $L_{2}$ are I-potent left (respectively, right) ideals of $S$, then $L_{1} \cup L_{2}$ is I-potent left (respectively, right) ideal of $S$.

Proof. If $L_{1}$ and $L_{2}$ are $I$-potent left ideals of $S$, then there exist positive integers $n_{1}, n_{2}$ such that $\left(L_{1}^{n_{1}}\right] \subseteq I$ and $\left(L_{2}^{n_{2}}\right] \subseteq I$, and hence $\left(\left(L_{1} \cup L_{2}\right)^{n_{1}+n_{2}}\right] \subseteq$ $I$. The second half of the result is proved similarly.

Theorem 5.2. Let $(S, \cdot, \leq)$ be an ordered semigroup having a kernel I. Any I-potent one-sided ideal of $S$ is contained in some I-potent two-sided ideal of $S$. 
Proof. Let $L$ be any $I$-potent left ideal of $S$; then $\left(L^{n}\right] \subseteq I$ for some positive integer $n$. If $(L S]$ is an $I$-potent two-sided ideal of $S$, then $L \cup(L S]$ is $I$-potent two-sided ideal of $S$ by Theorem 5.1, and hence the result follows. Indeed,

$$
\left((L S)^{n}\right]=(L(S L) \cdots(S L) S] \subseteq\left(L^{n} S\right] \subseteq(I S] \subseteq I .
$$

Thus $(L S]$ is an $I$-potent two-sided ideal of $S$ as required.

Definition. The union of all $I$-potent two-sided ideals of an ordered semigroup $(S, \cdot, \leq)$ having a kernel $I$ is called the radical of $S$.

Theorem 5.3. Let $(S, \cdot, \leq)$ be an ordered semigroup having a kernel $I$ and without a proper radical. Then every simple left ideal of $S$ is contained in a simple two-sided ideal of $S$.

Proof. Let $L$ be a simple left ideal of $S$. We have $L \cup(L S]$ is a two-sided ideal of $S$ containing $L$. We prove that $L \cup(L S]$ is simple. Let $M$ be a two-sided ideal of $S$ such that $I \subset M \subset L \cup(L S]$. Since $(M L]$ is a left ideal of $S$ such that $I \subseteq(M L] \subseteq L$, so $(M L]=I$ or $(M L]=L$. If $(M L]=I$, then

$$
(M M]=(M(L \cup L S)]=(M L \cup M L S] \subseteq I .
$$

This is a contradiction. If $(M L]=L$, then

$$
L \cup L S=(M L] \cup(M L] S \subseteq M .
$$

This is impossible. The proof is completed.

\section{References}

[1] G. Birkhoff, Lattice Theory, 25, Rhode Island, American Mathematical Society Colloquium Publ., Am. Math. Soc., Providence, 1984.

[2] N. Kehayopulu, On weakly prime ideals of ordered semigroups, Math. Japon. 35 (1990), no. 6, 1051-1056.

[3] N. Kehayopulu and S. Tsingelis, A note on groupoids-semigroups, Sci. Math. 3 (2000), no. 2, 251-255.

[4] - On kernels of ordered semigroups-a corrigendum, Commun. Korean Math. Soc. 28 (2013), no. 2, 225-229.

[5] M. Petrich, Introduction to Semigroups, Merrill, Columbus, 1973.

[6] Št. Schwarz, On semigroups having a kernel, Czechoslovak Math. J. 1(76) (1951), 229264.

Department of Mathematics

Faculty of Science

Khon Kaen University

Khon KaEn 40002, Thailand

E-mail address: thacha@kku.ac.th 